\title{
Estimulación temporal de nervio periférico en el dolor oncológico: opciones de oportunidad
} Temporary peripheral nerve stimulation in cancer pain:
opportunity options

\author{
M. L. Cánovas Martínez
}

Unidad del Dolor. Complejo Hospitalario Universitario de Ourense, España

La neuromudulación por estimulación de nervio periférico es una modalidad terapéutica del dolor de interés y evidencia creciente. Este estudio piloto pretende demostrar que la estimulación nerviosa periférica percutánea, utilizada de forma temporal, puede ser eficaz en el alivio de varios tipos de dolor prevalentes en la población oncológica: dolor agudo, dolor posamputación y dolor lumbar. Para ello implantaron, con control ecográfico y con una duración de 60 días, un electrodo en diferentes nervios, que posteriormente retiraron, consiguiendo en 7 de 12 pacientes un alivio mantenido de su dolor rebelde a múltiples tratamientos farmacológicos e intervencionistas (1).

Tres de estos casos demostraron como objetivo la estimulación de los nervios espinales proximales para tratar el dolor neuropático del tronco y el dolor radicular lumbar. Los cuatro casos restantes demostraron un objetivo exitoso que es la estimulación de otros nervios periféricos y del plexo braquial.

Si analizamos caso por caso, nos encontramos en primer lugar con una paciente que sufre un síndrome posmastectomía con neuralgia intercostobraquial de años de evolución.

El síndrome de dolor posmastectomía (SDPM) aparece recogido en la nueva clasificación ICD-11 (International Classification of Diseases $11^{\text {th }}$ Revision como un subgrupo de dolor crónico posquirúrgico. Waltho y Rockwell [2] han consensuado recientemente en un artículo de revisión lo que ellos denominan síndrome de dolor poscirugía mamaria (estos criterios han sido publicados en nuestra lengua en la RESED), que describen esta situación como un dolor que se produce después de cualquier cirugía de mama; es de, al menos, intensidad moderada, presenta características neuropáticas, se localiza en la mama, pared torácica, axila o brazo ipsilateral, tiene una duración mínima de 6 meses, ocurre al menos el $50 \%$ del tiempo y puede ser exacerbado por los movimientos de la cintura escapular. Es probablemente secundario a una lesión del nervio intercos- tobraquial o intercostales durante la disección a nivel axilar, lo que explica las características neuropáticas de este dolor. La incidencia documentada de SDPM varía según las publicaciones entre un 11 y un $57 \%$, bajando a un $5-10 \%$ en caso de que sea severo, destacando que hasta el $65 \%$ de los casos presentan características neuropáticas (3). Esta paciente fue tratada con fármacos considerados de primera línea para el tratamiento de este síndrome: gabapentina, pregabalina y amitriptilina; sin embargo, no se utilizaron fármacos de segunda línea como el parche de capsaicina al $8 \%$, que ha demostrado en este síndrome alivio de dolor mayor a las seis semanas conseguidas con la estimulación periférica tras la retirada de la misma (4). Tampoco fue tratada con técnicas intervencionistas como bloqueos paravertebrales o PECS block II (nervios pectorales, intercostobraquial, intercostales III-VI y nervio torácico largo] indicados en este tipo de dolor [5].

El segundo paciente presentaba una neuralgia posherpética, frecuente en el paciente oncológico, debido a la inmunosupresión y al tratamiento con quimioterapia y radioterapia [6]. Este paciente presentaba dolor severo y alodinia a pesar del tratamiento con fármacos de primera línea y con bloqueos intercostales seguidos de crioablación. La estimulación periférica del nervio espinal T7 produjo una analgesia de 12 meses tras la retirada del electrodo implantado durante 45 días. Otros tratamientos no utilizados en este paciente han mostrado eficacia en este cuadro de dolor: capsaicina al $8 \%$ (7) y radiofrecuencia pulsada del ganglio de la raíz dorsal torácica con alivio del dolor de meses de duración y de menor coste [8].

El tercer paciente presentaba una radiculopatía L2-L3, por un tumor del psoas, que respondió durante varias semanas al bloqueo del simpático lumbar a nivel L2-L3. El bloqueo neurolítico de la cadena simpática podría haber prolongado el tiempo de analgesia [9].

El cuarto paciente presentaba dolor secundario a metástasis con afectación C4-C6, que le ocasionaba un 
dolor severo que no respondió a tratamiento farmacológico ni a técnicas intervencionistas, como bloqueo del ramo medial de C4-C6, inyección de esteroides epidurales, infiltración glenohumeral, infiltración de la bursa subacromial y descompresión quirúrgica. La estimulación del nervio supraescapular, en este caso, supuso un alivio del dolor que se prolongó hasta los 18 meses de la extracción del electrodo. La paciente tuvo un bloqueo exitoso de la raíz afectada que duró solo 24 horas pero fue positivo; podría haberse intentando una radiofrecuencia pulsada de C5. La localización del dolor en el hombro y en la región dorsal hacen dudar de la larga mejoría con la estimulación del nervio supraescapular frente a estas técnicas.

La paciente 5 presentaba una radiculopatía C5-C8 secundaria a metástasis de un cáncer vaginal a nivel del plexo braquial. Previamente a la colocación del electrodo a nivel del plexo, a pesar de un alivio completo del dolor con el bloqueo del nervio supraescapular ipsilateral, no se probó la radiofrecuencia pulsada de dicho nervio (10).

El paciente 6 presentaba metástasis en el cuerpo vertebral de L5, secundario a adenocarcinoma de pulmón, que le ocasionó una radiculopatía L5-S1 tras resección quirúrgica del mismo. Con la estimulación periférica del nervio ciático se consiguió un alivio del dolor de seis meses de duración con mejoría funcional, que se limitó en el tiempo por el empeoramiento del paciente debido a la progresión del tumor. Creemos que antes de la implantación del estimulador deben intentarse medidas menos costosas y con eficacia demostrada en la radiculopatía posquirúrgica y producida por infiltración neoplásica. Parece razonable intentar en primer lugar una RF pulsada del ganglio de la raíz dorsal de L5 y S1, combinada con infiltración epidural transforaminal que puede complementarse, en algún momento de la evolución del proceso, con bloqueo ecoguiado del nervio ciático con anestésico local y corticoides para reducir el posible edema e inflamación acompañantes. Incluso plantear la colocación de un catéter, sobre el nervio, para administración continua de anestésico local a bajas dosis.

El paciente 7 presentó una resección de liposarcoma mixoide del muslo, región anterior izquierda que se presenta con dolor crónico rebelde a tratamiento farmacológico, dos años después de la resección. Se realiza bloqueo con esteroides de la rama cutánea anterior izquierda del nervio femoral.

Los síntomas de una neuropatía femoral incluyen dolor en la región inguinal que mejora y disestesias en la región anterior del muslo y anteromedial de la pierna. Los pacientes se quejan de dificultad para caminar o bloqueo subjetivo de la rodilla dependiendo de la severidad de la lesión.

Creemos que en este caso la posibilidad de implantar un sistema de estimulación periférica es una opción a considerar, dado que el paciente obtuvo una importante mejoría del dolor tras la inyección de esteroides sobre la rama cutánea anterior izquierda del nervio femoral (95\%). Sin embargo, no se especificó el tiempo de duración de este efecto analgésico del corticoide. Además de la técnica de bloqueo periférico, podrían considerarse las técnicas transforaminales y radiofrecuencia pulsada del GRD de L2, L3 y L4 (11).
El propósito de esta serie de casos fue demostrar dianas neuronales periféricas que podrían usarse de manera factible para tratar varios sindromes de dolor, prevalentes en la población oncológica, rebeldes a tratamiento farmacológico y que deberían ser rebeldes a otras técnicas intervencionistas previamente.

El bloqueo neural positivo para el alivio del dolor debería considerarse como paso previo a la implantación, puesto que dibuja el mapa analgésico esperable en los pacientes, con un proceso oncológico avanzado, como el paciente 6 cuya duración del efecto analgésico fue muy limitado debido a la progresión de la enfermedad.

\section{BIBLIOGRAFÍA}

1. Mainkar O, Sollo CA, Chen G, Legler A, Gulati A. Pilot Study in Temporary Pheripheral Nerve Stimulaction in Oncologic Pain. Neuromodulation. 2020;23(6):819-26. DOI: 10.1111/ ner.13139.

2. Waltho D, Rockwell G. Post-breast surgery pain syndrome: Establishing a consensus for the definition of post-mastectomy pain syndrome to provide a standardized clinical and research approach: a review of the literature and discussion. Can J Surg. 2016;59(5):342-50. DOI: 10.1503/ cjs.000716.

3. Mayo Moldes M, Fernández Rodríguez T, Illodo Miramontes G, Carregal Raño A, Goberna Iglesias MJ. Incidencia del síndrome posmastectomía. Análisis retrospectivo. Rev Soc Esp Dolor. 2020;27(4):246-51. DOI: 10.20986/ resed.2020.3797/2020.

4. Tait RC, Zoberi K, Ferguson M, Levenhagen K, Rowland K. Persistent Post-Mastectomy Pain: Risk Factors and Current Approaches to Treatment. J Pain. 2018:19(12):1367-83. DOI: 10.1016/j.jpain.2018.06.002.

5. Elzohry AAM, Elhamed MFA, Mahran MH. Post Mastectomy Pain is no Longer Nightmare. J Pain Manage Med. 2018;4(1):1-6.

6. Habel LA, Ray GT, Silverberg MJ, Horberg MA, Yawn BP, Castillo $A L$, et al. The epidemiology of herpes zoster in patients with newly diagnosed cancer. Cancer Epidemiol Biomarkers Prev. 2013;22(1):82-90. DOI: 10.1158/1055-9965.EPI$12-0815$.

7. Derry S, Rice ASC, Cole P, Tant T, Moore RA. Topical capsaicin (high concentration) for chronic neuropathic pain in adults. Cochrane Database Syst Rev. 2017;1(1):CD007393. DOI: 10.1002/14651858.CDO07393.pub4.

8. Ke M, Yinghui F, Yi J, Xeuhua H, Xiaoming L, Zhijun C, et al. Efficacy of pulsed radiofrequency in the treatment of thoracic postherpetic neuralgia from the angulus costae: a randomized, double-blinded, controlled trial. Pain Physician. 2013;16(1):15-25.

9. Plancarte R, Guajardo J. Efectividad de los procedimientos intervencionistas de la cadena simpática. Rev Arg Anest. 2005;63(6):807-12.

10. Gofeld M, Restrepo-Garces CE, Theodore BR, Faclier G. Pulsed radiofrequency of suprascapular nerve for chronic shoulder pain: a randomized double-blind active placebocontrolled study. Pain Practice. 2012;13(2):96-103. DOI: 10.1111/j.1533-2500.2012.00560.x.

11. Martínez F, Valverde A. Anatomía de los plexos lumbar y sacro En: Aliaga L. Anestesia Regional Hoy. 3. ${ }^{\text {a ed. Barcelo- }}$ na: Permanyer; 2006. p. 263-84. 